\title{
A MARKER-BASED EULERIAN-LAGRANGIAN METHOD FOR MULTIPHASE FLOW WITH SUPERSONIC COMBUSTION APPLICATIONS
}

\author{
XIAOFENG FAN \\ College of Aerospace Engineering, Nanjing University of Aeronautics and Astronautics, Nanjing, 210016, China \\ fanxiaofengnuaa@hotmail.com \\ JIANGFENG WANG \\ College of Aerospace Engineering, Nanjing University of Aeronautics and Astronautics, Nanjing, 210016, China \\ wangj@nuaa.edu.cn
}

Published 22 June 2016

\begin{abstract}
The atomization of liquid fuel is a kind of intricate dynamic process from continuous phase to discrete phase. Procedures of fuel spray in supersonic flow are modeled with an EulerianLagrangian computational fluid dynamics methodology. The method combines two distinct techniques and develops an integrated numerical simulation method to simulate the atomization processes. The traditional finite volume method based on stationary (Eulerian) Cartesian grid is used to resolve the flow field, and multi-component Navier-Stokes equations are adopted in present work, with accounting for the mass exchange and heat transfer occupied by vaporization process. The marker-based moving (Lagrangian) grid is utilized to depict the behavior of atomized liquid sprays injected into a gaseous environment, and discrete droplet model 13 is adopted. To verify the current approach, the proposed method is applied to simulate processes of liquid atomization in supersonic cross flow. Three classic breakup models, TAB model, wave model and K-H/R-T hybrid model, are discussed. The numerical results are compared with multiple perspectives quantitatively, including spray penetration height and droplet size distribution. In addition, the complex flow field structures induced by the presence of liquid spray are illustrated and discussed. It is validated that the maker-based Eulerian-Lagrangian method is effective and reliable.
\end{abstract}

Keywords: Eulerian-Lagrangian method; multiphase flow; supersonic crossflow.

\section{Introduction}

The atomization of liquid fuel is a kind of intricate dynamic process from continuous phase to discrete phase. The processes involve complex fluid phenomena such as combustion process, energy transport etc. Understanding atomization physics is important in many engineering applications, including scramjet and liquid rocket engine. In these propulsion systems, the combustion performance depends strongly on liquid

This is an Open Access article published by World Scientific Publishing Company. It is distributed under the terms of the Creative Commons Attribution 3.0 (CC-BY) License. Further distribution of this work is permitted, provided the original work is properly cited. 
atomization and the mixing process between free stream air and liquid jets. Thus, the study of the liquid fuel spray process in a high-speed cross flow is of great significance.

Several studies on liquid fuel spray were conducted in the past ${ }^{1-6}$. Many experimental researches on the droplets breakup process in the cross flow have been reported and it is well known that near-field jet breakup process involves three different regimes: liquid column, ligament and droplet regimes ${ }^{7}$. Although many experimental researches were reported so far, only limited numerical results have been reported regarding the highspeed cross flow. Theoretical and computational challenges involved in these processes are the spray atomization model selection.

Most of previous works on spray atomization model were focus on the liquid jets atomized in low-speed or subsonic crossflows and several mathematic models of atomization processes were proposed ${ }^{8}$. But in the case of supersonic flow, there still does not exists a comprehensive theory suitable to model all spray atomization phenomena, because of the difficulty of visualization of spray structure near the injector orifice.

Traditional methods use semi-empirical models to calculate the atomization processes. Reitz and Diwaker developed the formulation of the wave breakup model, and considered a cylindrical liquid jet of radius a penetrating through a circular orifice into a quiescent incompressible gas chamber ${ }^{9}$. O`Rourke and Amsden presented a numerical method, called the TAB (Taylor Analogy Breakup) model, for calculating drop aerodynamic breakup in engine sprays ${ }^{10}$. Patterson and Reitz developed the KelvinHelmholtz (K-H) and Rayleigh-Taylor (R-T) hybrid model, which allows unstable waves to grow simultaneously ${ }^{11}$.

In this paper, an integrated Eulerian-Lagrangian method is described and programmed into a multiphase simulation code, and then the proposed method is applied in simulation of liquid atomization in supersonic flow. And effects of liquid inertia, surface tension, aerodynamic forces on the liquid jet, as well as vaporization processes are taken into consideration. The results from three different secondary breakup models, TAB model, wave model and K-H/R-T hybrid model, are compared in multiple perspective, including spray penetration height and droplet size distribution. Numerical results are compared with those of existing numerical and experimental investigations conducted by other researchers. It is validated that the maker-based Eulerian-Lagrangian method is effective and reliable.

\section{Summary of Modeling Methods}

An integrated Eulerian-Lagrangian method is implemented for multiphase flow computations. The Finite Volume Method is applied to solve the gas flow field, and the discrete particle method is utilized to treat the behavior of spray, with accounting for the coupling with the unsteady evolution of the local flow and particle motion. The governing equations are multi-component three-dimension Navier-Stokes equations. The spatial distribution is discretized using Jameson scheme. In addition, the method is marched in time by a dual time-stepping for explicit Runge-Kutta integral method. 


\subsection{Gas phase models}

The governing equations for the gas phase in numerical simulation are multi-component three-dimension Navier-Stokes equations with liquid phase interaction source terms. The vector forms of the governing equations are given by,

$$
\frac{\partial \boldsymbol{Q}}{\partial t}+\frac{\partial \boldsymbol{E}}{\partial x}+\frac{\partial \boldsymbol{F}}{\partial y}+\frac{\partial \boldsymbol{G}}{\partial z}=\frac{\partial \boldsymbol{E}_{v}}{\partial x}+\frac{\partial \boldsymbol{F}_{v}}{\partial y}+\frac{\partial \boldsymbol{G}_{v}}{\partial z}+\boldsymbol{S}_{l},
$$

where $x, y, z$, and $t$ are the spatial coordinates and the evolution time, respectively. The vector of conservative variables $\boldsymbol{Q}$, vector of convective fluxes, $\boldsymbol{E}, \boldsymbol{F}$, and $\boldsymbol{G}$, vector of viscous fluxes, $\boldsymbol{E}_{v}, \boldsymbol{F}_{v}$, and $\boldsymbol{G}_{v}$, can refer to Ref. 12 for details. The source term $\boldsymbol{S}_{l}$, due to exchange terms between the gas and liquid spray particles are given by,

$$
\boldsymbol{S}_{l}=\left[\begin{array}{lllll}
\dot{\rho}_{i} & F_{x} & F_{y} & F_{z} & \dot{Q}
\end{array}\right]^{T},
$$

where $\dot{\rho}_{i}$ is the rate of change of species $i$ because of evaporating spray, the $F_{x}, F_{y}$, $F_{z}$ are the terms defining the $x, y$, and $z$ momentum exchanges induced by spray particles, the $\dot{Q}$ represents the work done by the particles to the gas and the heat flux into the control volume.

The analysis of gas-liquid two-phase flow is complicated by the need to account for the mass, momentum, and energy coupling between phases. To depict the behavior of atomized liquid sprays injecting into a gaseous environment, discrete droplet model $(\mathrm{DDM})^{13}$ is adopted in present work. The particle source in cell (PSIC) ${ }^{14}$ method is adopted to solve the source term $\boldsymbol{S}_{l}$. The droplets are regarded as sources of mass, momentum and energy to the conveying gaseous phase.

The source term in the continuity equation, $\dot{\rho}_{i}$, is given by,

$$
\dot{\rho}_{i}=-\frac{1}{\Omega \Delta t} \sum N_{n} \rho_{d} \frac{4}{3} \pi\left[\left(r^{n+1}\right)^{3}-\left(r^{n}\right)^{3}\right]
$$

where $\Omega$ is the volume of a computational cell, $\Delta t$ is the global physical time step, $N_{n}$ is the number of droplets in the control volume, $\rho_{d}$ is the density of droplets, $r$ is the radius of droplets. The superscript $n$ is previous time level and $n+1$ is new time level.

The momentum source terms $F_{x}, F_{y}$, and $F_{z}$ are given by,

$$
\begin{aligned}
& F_{x}=-\frac{1}{\Omega \Delta t} \sum N_{n} \rho_{d} \frac{4}{3} \pi\left[\left(r^{n+1}\right)^{3} u_{d}^{n+1}-\left(r^{n}\right)^{3} u_{d}^{n}\right] \\
& F_{y}=-\frac{1}{\Omega \Delta t} \sum N_{n} \rho_{d} \frac{4}{3} \pi\left[\left(r^{n+1}\right)^{3} v_{d}^{n+1}-\left(r^{n}\right)^{3} v_{d}^{n}\right], \\
& F_{z}=-\frac{1}{\Omega \Delta t} \sum N_{n} \rho_{d} \frac{4}{3} \pi\left[\left(r^{n+1}\right)^{3} w_{d}^{n+1}-\left(r^{n}\right)^{3} w_{d}^{n}\right]
\end{aligned}
$$

where $u_{d}, v_{d}, w_{d}$ denote the three components of droplets velocity in the Cartesian coordinate system. 
The energy source term $\dot{Q}$ is given by,

$$
\dot{Q}=-\frac{1}{\Omega \Delta t} \sum N_{n} \rho_{d} \frac{4}{3} \pi\left\{\begin{array}{l}
\left(r^{n+1}\right)^{3} e\left(T_{d}^{n+1}\right)-\left(r^{n}\right)^{3} e\left(T_{d}^{n}\right) \\
+\left(r^{n+1}\right)^{3}\left(u_{d}^{n+1}-u_{d}^{n}\right)\left(u_{d}^{n+1}-u^{n}\right) \\
+\frac{1}{2}\left[\left(r^{n+1}\right)^{3}-\left(r^{n}\right)^{3}\right]\left|u_{d}^{n+1}-u_{d}^{n}\right|^{2}
\end{array}\right\},
$$

where $T_{d}$ and $e\left(T_{d}\right)$ are the droplet temperature and internal energy, respectively, $u$ denotes the mean velocity of gaseous phase.

\subsection{Spray models}

In a Lagrangian reference frame, each computational particle represents a number of droplets with the same size, position, temperature and velocity. In other words, discrete particle method is adopted. The droplets locations, denoted by $\boldsymbol{x}_{d}$ in the Lagrangian frame, are updated from the velocity at its location, $\boldsymbol{v}_{d}$, in Eq. (6).

$$
\frac{d \boldsymbol{x}_{d}}{d t}=\boldsymbol{v}_{d}
$$

where subscript $d$ denotes the droplet.

The instantaneous particle velocity at arbitrary time is determined by solving the momentum equation.

$$
\frac{d \boldsymbol{v}_{d}}{d t}=\frac{3}{8} \frac{\rho}{\rho_{d}} \frac{\left|\boldsymbol{u}-\boldsymbol{v}_{d}\right|}{r}\left(\boldsymbol{u}-\boldsymbol{v}_{d}\right) C_{d}+\boldsymbol{g}-\frac{\nabla p}{\rho_{d}}
$$

In Eq. (7), $\boldsymbol{g}$ is standard gravity. The drag coefficient is determined by

$$
C_{d}=\left\{\begin{array}{cc}
\frac{24}{\operatorname{Re}_{d}}\left(1+1 / 6 \mathrm{Re}_{d}^{2 / 3}\right) & \mathrm{Re}_{d}<1000 \\
0.424 & \operatorname{Re}_{d} \geq 1000
\end{array},\right.
$$

the liquid Reynolds number $\mathrm{Re}_{d}$ in Eq. (8) is given by,

$$
\operatorname{Re}_{d}=\frac{2 \rho\left|\boldsymbol{u}-\boldsymbol{v}_{d}\right| r}{\mu_{d}} .
$$

\subsection{Vaporization Model}

Evaporating liquid droplets on a hot surface remove a large amount of heat through the latent heat contribution. Thus, the expansion of droplet due to the increase in droplet temperature at the overall process of evaporation is very significant. The variation of droplet diameter during evaporation is given by the following equation ${ }^{15}$, 


$$
\frac{d r}{d t}=-\frac{(\rho D)_{d}(\widehat{T})}{2 \rho_{d} r} \frac{Y_{1 s}-Y_{1}}{1-Y_{1 s}} S h_{d} .
$$

In Eq. (10), $\rho D$ is diffusion coefficient of vapor in gaseous phase and given by,

$$
(\rho D)_{d}=\rho_{\text {air }} D_{1} T^{D_{2}}
$$

where $D_{1}$ and $D_{2}$ are physical parameters of fuel. $S h_{d}$ is the Sherwood number, namely,

$$
S h_{d}=\left(2+0.6 \mathrm{Re}_{d}^{1 / 2} S c_{d}^{1 / 3}\right) \frac{\ln \left(1+B_{m}\right)}{B_{m}},
$$

where $B_{m}$ is the Spalding mass transfer number, $S c_{d}$ is the droplet Schmidt number, are given by,

$$
B_{m}=\frac{Y_{1 s}-Y_{1}}{1-Y_{1 s}}, \quad S c_{d}=\frac{\mu_{d}(\widehat{T})}{(\rho D)_{d}(\widehat{T})} .
$$

In Eq. (13), $Y$ is mass fraction of fuel vapor, $\mu_{d}$ is viscosity coefficient of fuel, subscript $l s$ and $l$ denote the surface of the drop and the drop locations in the Lagrangian frame, respectively. The weighted gas temperature $\widehat{T}$ is given by the two third law, namely,

$$
\widehat{T}=\left(T+2 T_{d}\right) / 3 .
$$

For simplicity, assume that heat transfer which caused by thermal radiation can be neglected, hear conduction is the only way to transfer heat. To estimate the convective heating rate from the ambience to a droplet, consider the following pseudo-steady energy conservation equation in the vapor phase:

$$
\rho_{d} \frac{4}{3} \pi r^{3} c_{l}\left(T_{d}\right) \dot{T}_{d}-\rho_{d} 4 \pi r^{2} \dot{r} L\left(T_{d}\right)=4 \pi r^{2} Q_{d}
$$

In the above equation, $c_{l}$ is the specific heat of the fuel vapor, and $L$ is the vaporization latent heat. $Q_{d}$ is the convective heating rate, defined as,

$$
Q_{d}=\frac{\kappa_{d}(\widehat{T})\left(T-T_{d}\right)}{2 r} N u_{d} .
$$

In Eq. (16), $\kappa_{d}$ is gas phase thermal conductivity, $N u_{d}$ is Nusselt number, for the situations with forced convection, defined as,

$$
N u_{d}=\left(2+0.6 \operatorname{Re}_{d}^{1 / 2} \operatorname{Pr}_{d}^{1 / 3}\right) \frac{\ln \left(1+B_{h}\right)}{B_{h}} .
$$

where $B_{h}$ is the Spalding heat transfer number, $P r_{d}$ is the Prandtl number for droplet, are given by, 


$$
B_{h}=\frac{c_{p}(T)\left(T-T_{d}\right)}{L\left(T_{d}\right)}, \quad \operatorname{Pr}_{d}=\frac{m_{d}(\widehat{T}) c_{p}(\widehat{T})}{\kappa_{d}(\widehat{T})},
$$

where $c_{p}$ is the specific heat of the mixture gas at constant pressure. Spalding vaporization model postulates Lewis number is 1 , namely, $L e=S c / \operatorname{Pr}=1.0$. Thus $B_{h}=B_{m}$.

\subsection{Breakup Model}

Typically fuel injection is handled using a blob injection model and due to the interaction with the environment the computational parcels are subject to surface instabilities ${ }^{6}$. This section will describe three types of the typical breakup models in detail.

\subsubsection{TAB Model}

Taylor Analogy Breakup (TAB) model is based on an analogy between an oscillating and distorting droplet, and a spring mass system. The restoring force of the spring corresponds to the surface tension forces, external forces on the mass corresponds to the aerodynamic drag forces, and the damping force is represented by the viscosity of the liquid. The formulation of the classical TAB model is given below,

$$
\ddot{\vartheta}=\frac{2}{3} \frac{\rho}{\rho_{d}} \frac{\left|\vec{u}+\vec{u}^{\prime}-\vec{v}_{d}\right|^{2}}{r^{2}}-\frac{8 \sigma_{d}}{\rho_{d} r^{3}} \vartheta-\frac{5 \mu_{d}}{\rho_{d} r^{2}} \dot{\vartheta},
$$

where $\vartheta$ is the droplet displacement from equilibrium and with breakup occurring if and only if $\vartheta>1$.

The prediction of droplet sizes after breakup is based upon an energy conservation analysis. In the analysis, a balance of the energy of the parent drop with the energies of the newly generated drops after breakup carried out to yield,

$$
\frac{r_{0}}{r_{32}}=1+\frac{8 K}{20}+\frac{\rho_{d} r_{0}^{3}}{\sigma_{d}} \ddot{\vartheta}\left(\frac{6 K-5}{120}\right),
$$

where $r_{0}$ is parent droplet radius, $r_{32}$ is the radius of newly generated droplets, and $K$ a constant that determined by experiments, $K=10 / 3$ by default.

\subsubsection{Retiz Wave Model}

The formulation of the Wave Breakup Model developed primarily by Reitz and Diwaker considers a cylindrical liquid jet of radius a penetrating through a circular orifice into a quiescent incompressible gas chamber ${ }^{16}$. A simplification is made by Reitz ${ }^{17}$ by generating curve fits of numerical solutions to the dispersion equation which the following expressions were obtained for the maximum growth rate $\Omega_{K H}$ and the corresponding wavelength $\Lambda_{K H}$, 


$$
\begin{aligned}
& \Lambda_{K H}=\frac{9.02 r_{0}(1+0.45 \sqrt{Z})\left(1+0.4(T)^{0.7}\right)}{\left(1+0.865 W e^{1.67}\right)^{0.6}}, \\
& \Omega_{K H}=\frac{0.34+0.385 W e^{1.5}}{(1+Z)\left(1+1.4 T^{0.6}\right)} \sqrt{\frac{\sigma_{d}}{\rho_{d} r_{0}^{3}}}
\end{aligned}
$$

where,

$$
Z=\sqrt{W e_{l}} / \operatorname{Re}_{l}, W e=\rho \vec{u}_{r}^{2} r / \sigma_{d}, W e_{l}=\rho_{d} \vec{u}_{r}^{2} r / \sigma_{d}, T=\sqrt{W e},
$$

where $Z$ is Ohnesorge number, $W e$ is Weber number.

Droplet size is estimated assuming a linear relationship between the resulting droplet radius $r$, and the wavelength $\Lambda_{K H}$ of the most unstable surface disturbance (where $B_{0}$ is $0.61)$

$$
r=B_{0} \Lambda_{K H}
$$

Variations of parent droplet radius $r_{0}$ is given by,

$$
\frac{d r_{0}}{d t}=-\frac{r_{0}-r}{\tau_{K H}}, \tau_{K H}=\frac{3.788 B_{1} r_{0}}{\Omega_{K H} \Lambda_{K H}},
$$

where the constant $B_{1}$ accounts for the nozzle geometry.

\subsection{3. $K-H / R-T$ hybrid model}

Original Rayleigh-Taylor model is formulated based on theoretical analysis of the stability of liquid-gas interfaces caused by accelerations at a normal direction to the plane. Chang ${ }^{18}$ assumed a linearized disturbance growth rate and negligible viscosity, the frequency and wavelength of the fastest growing waves are derived as,

$$
\begin{aligned}
& \Lambda_{R T}=2 \pi \sqrt{\frac{3 \sigma_{d}}{a_{d}\left(\rho-\rho_{d}\right)}} \\
& \Omega_{R T}=\sqrt{\frac{2 a_{d}}{3} \frac{\rho-\rho_{d}}{\rho+\rho_{d}}}\left[\frac{a_{d}\left(\rho-\rho_{d}\right)}{3 \sigma_{d}}\right]^{1 / 4},
\end{aligned}
$$

where $a_{d}$ is acceleration of droplets in the gaseous phase. In this model it is assumed that the liquid droplets are accelerated by frag forces only, and its mathematical expression is given by,

$$
a_{d}=\frac{3}{8} C_{d} \frac{\rho u_{r}^{2}}{\rho_{d} r},
$$

where $u_{r}$ is the relative velocity between the droplet and gas, $r$ is the droplet radius. 
The breakup time is given by,

$$
\tau_{R T}=\sqrt{\frac{\sqrt{\sigma_{d}}\left(\rho+\rho_{d}\right)}{2}\left(\frac{3}{a_{d}\left(\rho-\rho_{d}\right)}\right)^{3 / 2}} .
$$

The breakup process is only allowed when $\Lambda_{R T}<r_{o}$ and the radius of product droplets is given by,

$$
r=\frac{1}{2} \Lambda_{R T}
$$

Patterson ${ }^{11}$ presented a new breakup model, the R-T model is used in combination of the Retiz wave model (K-H model) as a hybrid K-H/R-T model to describe secondary droplet breakup. The R-T and K-H models are implemented in competing manners, such that the droplet breaks up by the model that predicts a shorter breakup time.

\section{Model Validation and Verification}

To validate the simulation approach, the structures of water jets injecting into an $\mathrm{M}=1.94$ crossflow are studied numerically, and all test condition and geometrical parameters in numerical calculation can consult Ref. 11. The spatial distribution was discretized using Jameson scheme, and the solutions are advanced in time with a Runge-Kutta scheme, and a dual-time step sub-iteration scheme is used to compute unsteady flows. In addition, physical properties of water (density, surface tension, viscosity coefficient, critical temperature, etc.) are brought from experimental data ${ }^{20}$.

\subsection{Breakup Model}

The main focus of this section is to study the variation of specific physical parameters, such as penetration height, droplet size (Sauter Mean Diameter, SMD), etc., with different breakup models. Thus, mass exchange and heat transfer occupied by vaporization process is not considered which will be the subject of next section.

The jet-to-air momentum flux ratio, $q_{0}$, is defined as,

$$
q_{0}=\rho_{I n} v_{I n}^{2} / \rho_{\infty} v_{\infty}^{2},
$$

where subscript In denotes injector.

The Sauter mean diameter is given by,

$$
S M D=\sum N_{n}\left(2 r_{d}\right)^{3} / \sum N_{n}\left(2 r_{d}\right)^{2} .
$$

Test conditions are summarized in Table 1. The calculation domain has a height of $127 \mathrm{~mm}$, width of $152 \mathrm{~mm}$, and length of $600 \mathrm{~mm}$. A spray injector is located at $139 \mathrm{~mm}$ from the leading edge of the calculation domain, with a radius of $0.5 \mathrm{~mm}$. 
Table 1 Conditions for spray atomization in supersonic crossflow

\begin{tabular}{|c|c|c|c|c|c|}
\hline \multirow{2}{*}{ No. } & \multicolumn{2}{|c|}{ Incoming air conditions } & \multicolumn{2}{|c|}{ Injection conditions } & \multirow{2}{*}{ Breakup model } \\
\hline & Pressure $(\mathrm{KPa})$ & Temperature(K) & $q_{0}$ & Temperature(K) & \\
\hline 1 & \multirow{3}{*}{29.00} & \multirow{3}{*}{304.1} & \multirow{3}{*}{10.0} & \multirow{3}{*}{298.15} & TAB model \\
\hline 2 & & & & & Retiz wave model \\
\hline 3 & & & & & K-H/R-T hybrid model \\
\hline
\end{tabular}

Fig. 1a, b, and c show the numerical predicted two-dimensional droplet distributions on the center plane of the $y$-axis directly extracted from the three-dimensional domain particle field. In addition, the results of the penetration height calculated from experimental correlation function, which is developed by $\mathrm{Liu}^{21}$, are also plotted to compare to numerical simulations. Liu proposes the correlation function, using schlieren method, which is given by the following equation,

$$
\frac{h}{d}=3.14 q_{0}^{0.29}\left(\frac{x}{d}\right)^{0.38},
$$

where $h$ is penetration height, $d$ is nozzle orifice diameter. The results of three breakup model are compared with each other in Fig. 1, and display that the TAB model and the K$\mathrm{H} / \mathrm{R}-\mathrm{T}$ model are similar to the penetration height, while, in contrast, wave model shows a higher penetration height which outclass that of experimental data. In addition, the spray flow with the TAB model shows a different particle distribution and droplet distribution compared to the K-H/R-T model. In the case of the TAB, the minimum droplet size appears at the bottom of the flow field, where the gaseous phase velocity appears to be relatively small, while, the K-H/R-T model shows a wider and higher droplet distribution, which agrees well with the experimental phenomenon.

Fig. 1d illustrates the comparisons of the SMD distribution along the z-axis at the center plane of $y$-axis and at the position of $x=50 \mathrm{~mm}$ from the origin of the injector. With the wave model, the droplet size distribution is monotonously increasing. When the TAB model is applied, the distribution is changed showing that the distribution trends become closer to the experimental results, but the size of droplets are smaller than experimental data. Even though there are still distinguishable differences on the distribution trends, the K-H/R-T model predicts more accuracy in terms of the range of the droplet sizes. 


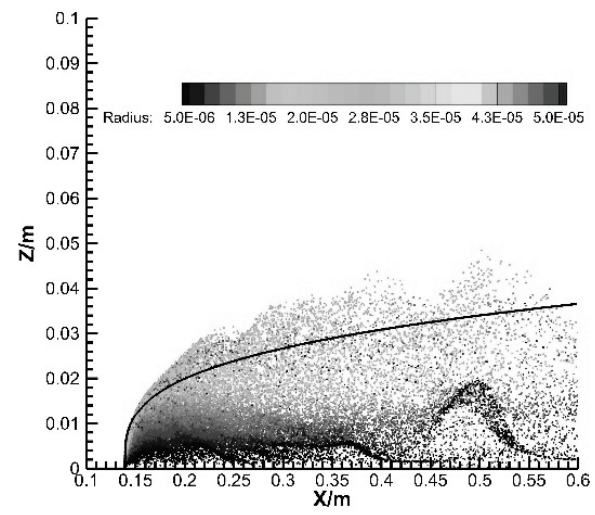

(a) TAB model

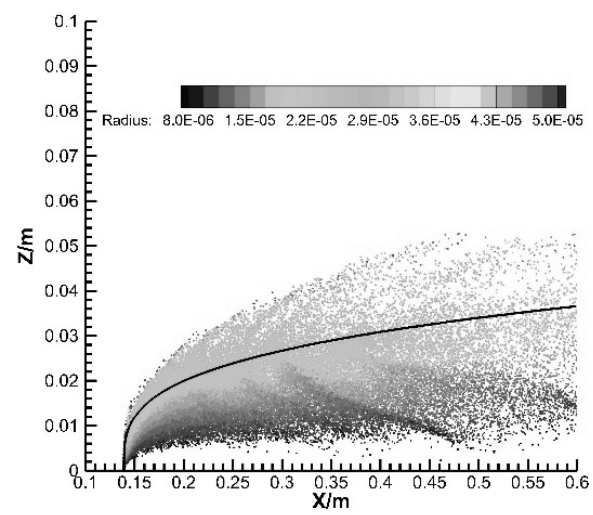

(c) K-H/R-T hybrid model

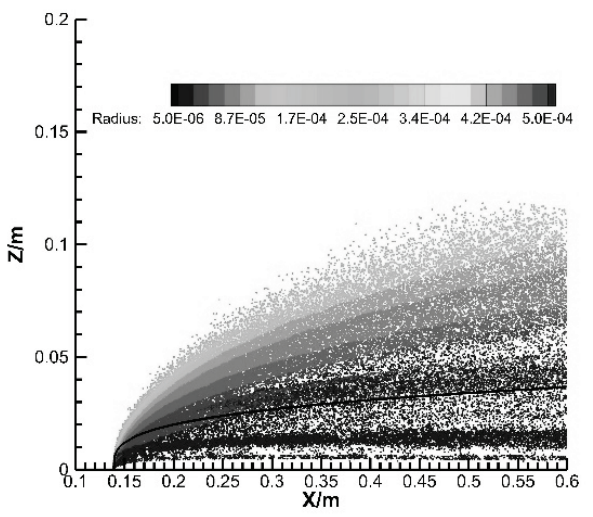

(b) Retiz wave model

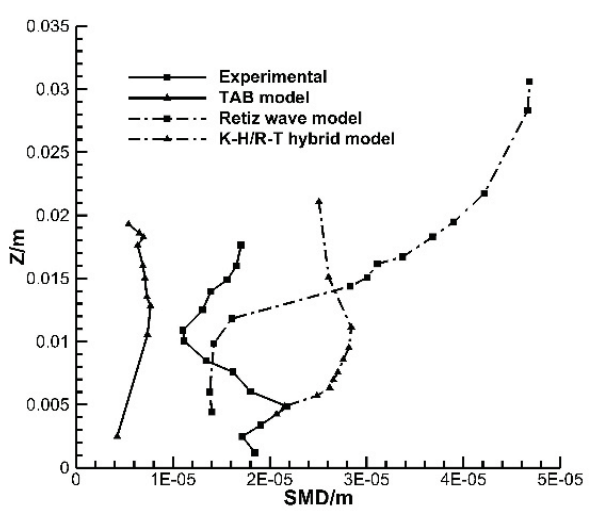

(d) SMD distribution of droplets

Fig. 1 Comparisons of spray penetration heights and droplet sizes.

\subsection{Vaporization Model}

Droplet vaporization has great effects on the processes of spray atomization. The process of droplet evaporation contains heat transfer and mass transfer. In this section, effects of vaporization processes on droplet size, droplet distribution, and spray structure have been discussed in detail. Based on previous observations, the K-H/R-T hybrid model seems to be a suitable model in a high-speed cross flow situation. Thus, the K-H/R-T hybrid model is adopted in present case. In addition, the other conditions, such as, Incoming air conditions, injection conditions, geometrical parameters, etc., remain unchanged.

The environmental temperature influences the heat transfer during the droplet vaporization. So the variety of the mass fraction field of $\mathrm{H}_{2} \mathrm{O}$ during the vaporization process is shown in Fig. 2. And the relevant physical flow times are $30 \mu \mathrm{s}$ and $105 \mu \mathrm{s}$, respectively. During the droplet evaporating, the water-vapor due to droplet vaporization 
is diffused and mixed with gas continuously. Fig. 3 Shows the comparisons of the SMD distribution along the $z$-axis at the center plane of $y$-axis and $x=50 \mathrm{~mm}$ from the injector nozzle. Fig. 3a displays the droplet size distributions at the start of fuel injection, namely, flow time is $30 \mu \mathrm{s}$. When the vaporization model is applied, the droplet size is small compared with the no vaporization model case. This is due to the effects of temperature gradient makes the heat transfer between ambient and droplets. Based on the observations of Fig. 3, drops with small size are shed along the flow direction first, and ligaments with bigger size reach the same position need more time. Referring to Fig. 3b, the case with vaporization model predicts more accuracy in terms of the range of the droplet sizes. However, there are still discernible differences on the distribution trends.
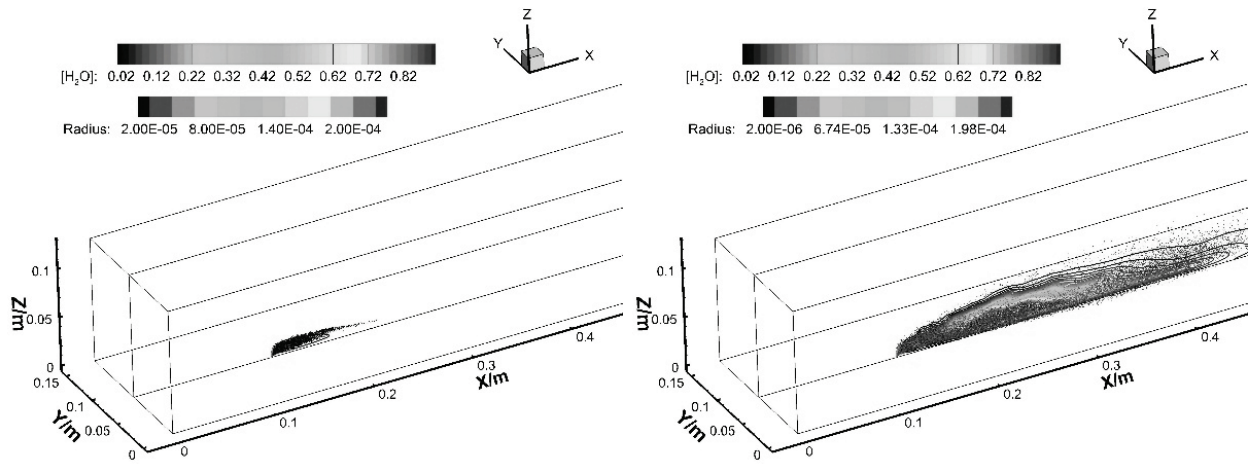

(a) $30 \mu \mathrm{s}$

(b) $105 \mu s$

Fig. 2 Variety of the mass fraction field of $\mathrm{H}_{2} \mathrm{O}$.

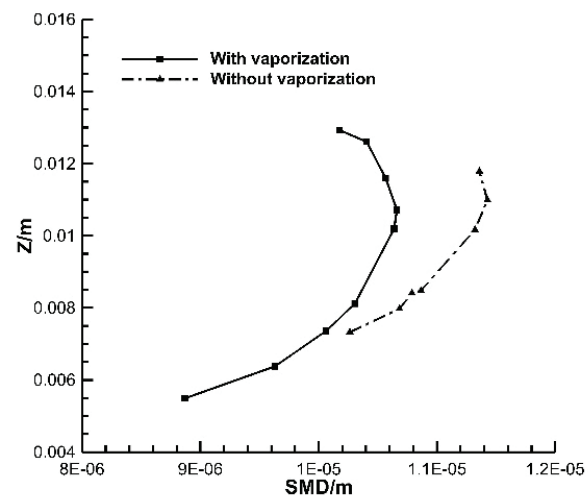

(a) $30 \mu \mathrm{s}$

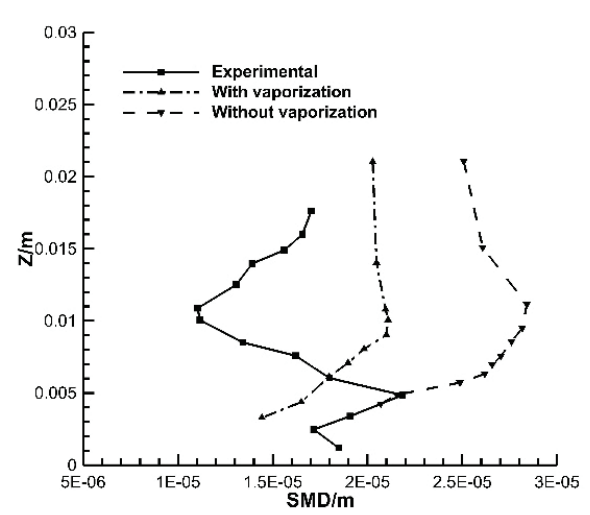

(b) $105 \mu s$

Fig. 3 SMD distribution of droplets along the $z$-axis at $y=$ center and $x=50 \mathrm{~mm}$ from the injector nozzle. 


\section{Conclusions}

An integrated Eulerian-Lagrangian method is described. To verify the current approach, the proposed method is applied to simulate processes of liquid atomization in supersonic cross flow. Three classic breakup models, TAB model, wave model and K-H/R-T hybrid model, are discussed. By comparing with experimental data, the simulation results of the $\mathrm{K}-\mathrm{H} / \mathrm{R}-\mathrm{T}$ hybrid model are more accurate than that of remaining two models in terms of spray penetration height and droplet size distribution.

In addition, numerical simulations of unsteady flow due to gas-liquid interaction in supersonic cross flows are performed, and the effects of vaporization processes are taken into consideration. A commonly used vaporization model proposed by Spalding is adopted in present work. During the droplet evaporating, the water-vapor is diffused and mixed with gas continuously which directly affects the complex flow field structure induced by the presence of liquid spray, including mass faction field, density field, etc. A comparison is made between experimental data and numerical results, which shows that the case with vaporization model predicts more accuracy in terms of the range of the droplet sizes.

Detailed numerical simulations are able to provide comprehensive insight into the mechanism of liquid jet in supersonic cross flow. A more detailed research on subprocedures of liquid atomization, such as drop collision and coalescence, may be required.

\section{References}

1. S. I. Kyoung, K. C. Lin, M. C. Lai, Spray atomization of liquid jet in supersonic cross flows, Tech. Rep. 0732, (2005).

2. G. Yu, J.G. Li and J.R. Zhao, Proc. Combust. Inst. 30, 2859, (2005).

3. K. Brinckman, A. Hosangadi and V. Ahuja, et al. AIAA Journal, (2000).

4. M. Pai, I. Bermejo-Moreno, O. Desjardins, Am. Phys. Soc. (2009).

5. P. Khare, D. Ma, X. Chen and V. Yang. Phenomenology of Secondary Breakup of Newtonian Liquid Droplets. (50th AIAA Aerospace Sciences Meeting, Nashville, Tennessee. 2012).

6. L. Bravo and C. Kweon, A Review on Liquid Spray Models for Diesel Engine Computational Analysis, Tech. Rep. 6932, Army Research Laboratory (2014).

7. P.K Wu, K.A. Kirkendal, R.P. Fuller and A. S. Nejad, J. Propuls. Power 13, 64, (1997).

8. S. Yang, J. Le, W. He, AIAA Journal, (2000).

9. Reitz R D, At. Spray Technol. 3, 309, (1987).

10. P. J. O'Rourke and A. A. Amsden, The TAB method for numerical calculation of spray droplet breakup, (International Fuels and Lubricants Meeting and Exposition, Toronto, 1987).

11. M. Patterson, Modeling the effects of fuel injection characteristics on diesel combustion and emissions, PhD thesis, University of Wisconsin-Madison (1997).

12. P. A. Gnoffo, R. N. Gupta and J. L. Shinn, Conservation equations and physical models for hypersonic air flows in thermal and chemical nonequilibrium. STI/Recon Tech. Rep. 89: 16115, NASA (1989).

13. J. K. Dukowicz, J. Comput. Phys. 35, 229, (1980).

14. C. T. Crowe, M. P. Sharma, D. E. Stock, J. of Fluids Eng. 99, 325 (1977).

15. Spalding D. Symp.on Combust. Combust. Inst., (1953). 
16. Reitz, R. and R. Diwakar, Effect of Drop Breakup on Fuel Sprays, SAE Tech. Pep. 860469, (1986).

17. R. Reitz, Spray Technology Short Course, (Pittsburgh, PA, 1996).

18. S. K. Chang. Hydrodynamics of liquid jet sprays. PhD thesis, University of WisconsinMadison (1991).

19. K. C. Lin, P. J. Kennedy and T. A. Jackson. Structures of water jets in a Mach 1.94 supersonic crossflow. (42nd AIAA aerospace sciences meeting and exhibit, Reno, 2004).

20. D. R. Lide, CRC Handbook of Chemistry and Physics, 88th edn. (CRC Press/Taylor and Francis, Boca Raton, FL, 2009)

21. J. Liu, Numerical and experimental investigation of fuel spray in supersonic cross flow, $\mathrm{PhD}$ thesis, Beihang University (2009). 